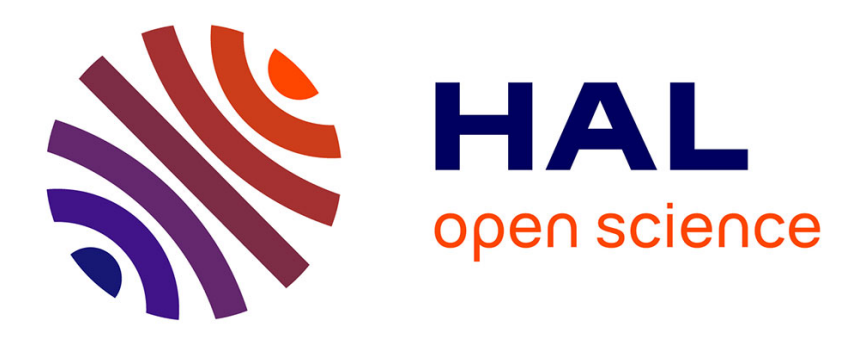

\title{
Properties of a pinned electron solid on a liquid helium film.
}

\author{
A. Dahm, H. Jiang
}

\section{To cite this version:}

A. Dahm, H. Jiang. Properties of a pinned electron solid on a liquid helium film.. Journal de Physique IV Proceedings, 1993, 03 (C2), pp.C2-39-C2-43. 10.1051/jp4:1993207 . jpa-00251576

\section{HAL Id: jpa-00251576 https://hal.science/jpa-00251576}

Submitted on 1 Jan 1993

HAL is a multi-disciplinary open access archive for the deposit and dissemination of scientific research documents, whether they are published or not. The documents may come from teaching and research institutions in France or abroad, or from public or private research centers.
L'archive ouverte pluridisciplinaire HAL, est destinée au dépôt et à la diffusion de documents scientifiques de niveau recherche, publiés ou non, émanant des établissements d'enseignement et de recherche français ou étrangers, des laboratoires publics ou privés. 


\title{
Properties of a pinned electron solid on a liquid helium film ${ }^{(1)}$
}

\author{
A.J. DAHM and H.W. JIANG*
}

Department of Physics, Case Western Reserve University, Cleveland, OH 44106, U.S.A. * Department of Physics, University of California at Los Angeles, Los Angeles, CA 97124, U.S.A.

\begin{abstract}
The conduction and noise properties of a classical electron solid pinned by varlations in the image potential of a glass substrate underneath a helium film supporting the electrons are presented. These properties include a nonlinear resistivity, a frequency-dependent complex impedance, $f^{-\alpha}$ noise and an abrupt increase in broad-band noise when the electron solid becomes depinned. The results of this study are compared with data on quantum electrons solids in heterostructures and MOSFETs.
\end{abstract}

\section{Introduction.}

Electrons on a liquid helium surface form a uniquely simple, two-dimensional system. When the helium consists of a thin film on a dielectric substrate, variations in the image potential of the electrons due to substrate roughness leads to a random lateral potential for the electrons. For thin films this "pinning potential" is sufficiently strong to pin the solid phase of the electron system. The conduction properties of this electron solid mimic those of sliding charge-density waves [1].

Kajita [2] was the first to observe non-linear I-V characteristics for electrons on a helium film supported by a hydrogen substrate. He suggested that this non-linearity resulted from the pinning of the electron crystal. Later Jiang and Dahm [3] made a more extensive study of the conduction and noise properties of this system using a glass substrate and verified that the system was indeed a pinned electron solid. Motivated by this work, measurements of the conduction properties of two-dimensional electrons have been adapted to identify the solid phase of the electron system in heterostructures [4-6] and silicon MOSFETS [7]. It is our purpose here to review the conduction and noise properties of the pinned electron solid on a helium film and compare these results with measurements in quantum systems.

We point out some differences between the electrons on a helium film system and electrons in semiconductors. A direct electrical contact cannot be made to electrons on helium. Thus, a dc voltage cannot be applied to this system. Secondly, in the stripline geometry we employ the current profile is trapezoidal across the length of the sample. Finally, our measurements are made on a classical system at temperatures relatively close to the melting point. Thermal excitation may play a role in the conduction process at low excitation voltages.

\section{Results.}

Our system consisted of a helium film supported by a $160 \mu \mathrm{m}$ thick cover glass. Two $1 \mathrm{~cm}$ square electrodes were evaporated on the underside of the glass. The electron layer above the helium film and the metallic electrodes formed an electrical stripline. Measurements were made by passing an ac current through the electron layer and measuring the real and imaginary parts of the impedance [8]. A constant voltage source was used. The applied field is approximately equal to the excitation voltage, $V_{o x}$ divided by one $\mathrm{cm}$. 


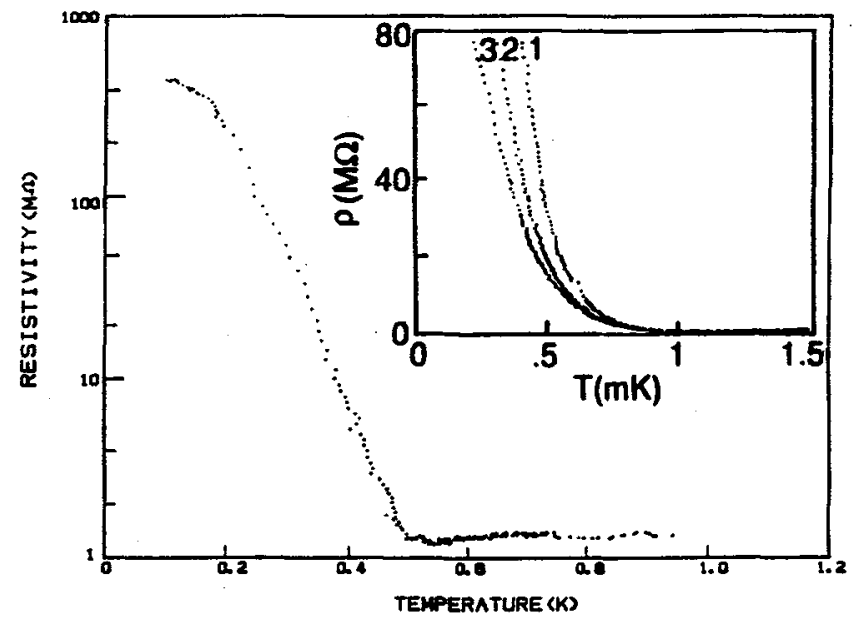

Fig. 1. Resistivity vs temperature: $n=2 \times 10^{9} \mathrm{~cm}^{-2}, t=380 \mathrm{~A}$, and $\mathrm{f}=100 \mathrm{~Hz}$ Main graph: $V_{e x}=2 \mathrm{mV}$. Inset: $V_{o x}=$ (1) $19 \mathrm{mV}$, (2) $28 \mathrm{mV}$, and (3) $37 \mathrm{mV}$.

The resistivity of the two-dimensional electron layer as a function of temperature is presented in Fig. 1. In the fluid phase the resistivity is nearly independent of temperature and is determined by electron scattering from helium surface ripplons and substrate potentials. The resistivity varles as $p \sim\left(-\gamma T / T_{c}\right)$ in the solid phase, where $\gamma$ is a constant. This dependence may reflect the thermal mean square deviation of the electrons from their lattice sites, $\left\langle x^{2}\right\rangle \sim T$. The linear scale for $\rho$ shown in the inset shows a smooth varlation with temperature through the melting point. We define the melting temperature as the intersection of the two segments of the resistivity curve plotted on a logarithmic scale. This value is consistent with the

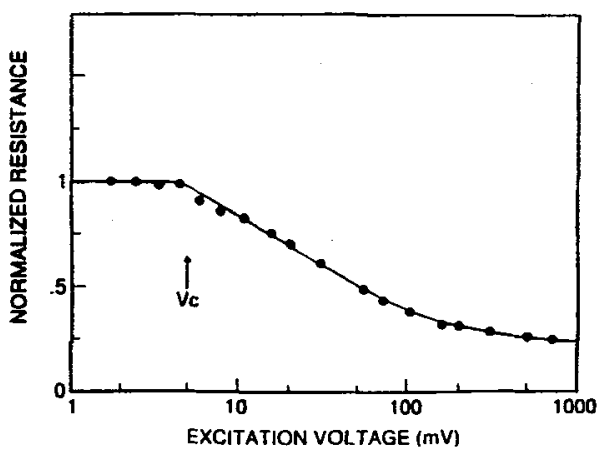

Fig. 2. Resistivity vs. excitation voltage: $n=5 \times 10^{9} \mathrm{~cm}^{-2}, t=320 \mathrm{~A}$, $f=1 \mathrm{kHz}$, and $\mathrm{T}=690 \mathrm{mK}$.

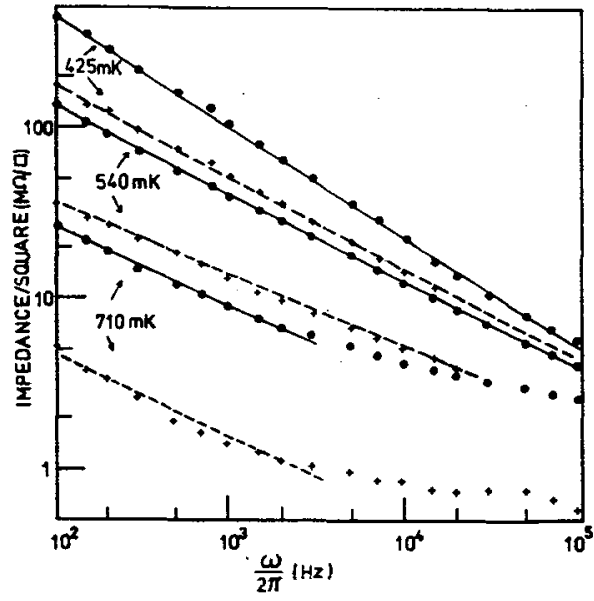

Fig. 3. Real and imaginary parts of the impedance. O resistivity; + reactance per square; $\mathrm{n}=3.8 \times 10^{9} \mathrm{~cm}^{-2}$. $t=340 \mathrm{~A}$ and $\mathrm{V}_{\mathrm{ox}}=20 \mathrm{mV}$. 


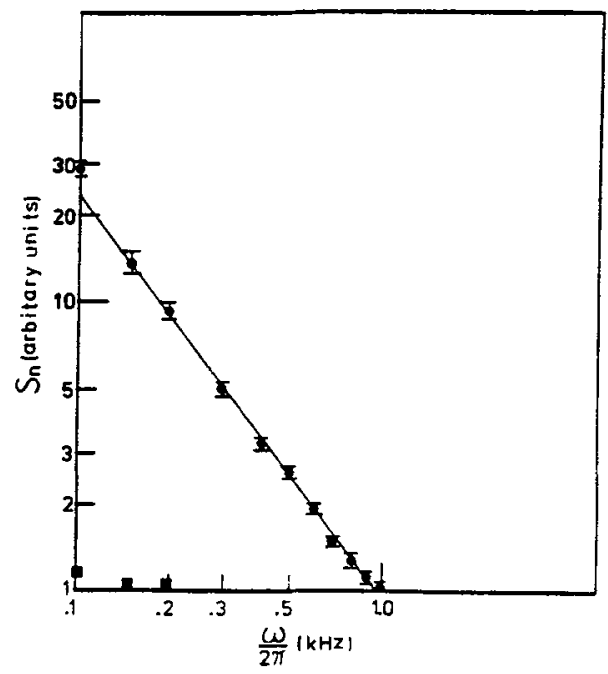

Fig. 4 Noise amplitude vs frequency: $\mathrm{n}=10^{\mathrm{io}} \mathrm{cm}^{-2}, \mathrm{t}=240 \mathrm{~A}, \mathrm{~T}=200 \mathrm{mK}$, and $V=35 \mathrm{mV}$. The filled squares represent the instrumental noise.

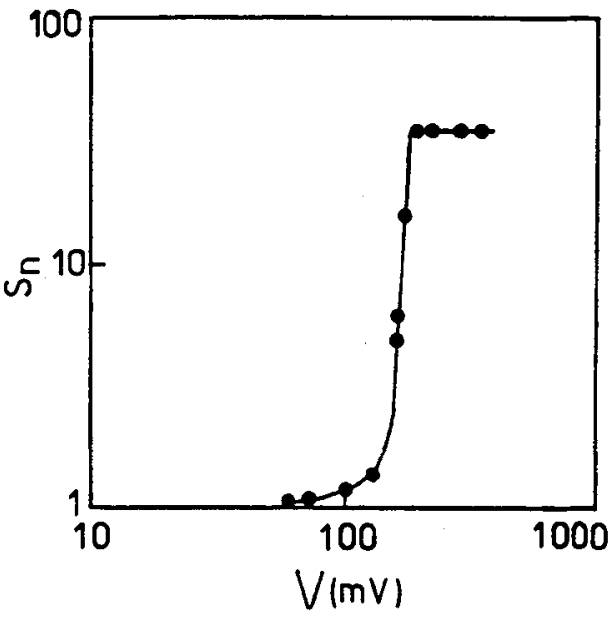

Fig. 5, Broad-band noise vs excitation voltage: $n=5 \times 10^{9} \mathrm{~cm}^{-2}, t=320 \mathrm{~A}$, $T=50 \mathrm{mK}$ and $\mathrm{f}=1 \mathrm{kHz}$.

theoretical value determined from the Kosterlitz-Thouless melting theory for a Coulomb interaction screened by a dielectric substrate $[9,10]$.

The I-V characteristics of the system are non-linear. In Fig. 2 we plot the normalized ac resistance as a function of excitation voltage. The resistance is a smooth function of the excitation voltage with a decrease setting in at a critical voltage $V_{c}$. Above a second critical voltage $V_{N}$, which is $i l l$ defined on a resistance plot, the resistance again becomes independent of excitation voltage.

The frequency dependence of the real and imaginary parts of the impedance per square are shown in Fig. 3. At low frequencies these vary as $\omega^{-\eta}$, where $\eta$ is temperature dependent. For this sample, $\eta$ takes on the values 0.63 and 0.46 respectively at 425 and $710 \mathrm{mK}$.

The noise characteristics are shown in Fig. 4. Between the critical voltages $V_{c}$ and $V_{N}$ the nolse varies as $f^{-\alpha}$, where $\alpha=1.4 \pm 0.1$. At voltages above $V_{N}$ the broad-band nolse increases abruptly by a factor of 30 .

In the weak-coupling limit a charge-density wave has phase coherence over a distance $L$. The dielectric screening of the electrons can be treated by placing an image charge a distance $2 t$ beneath each electron. Here $t$ is the helium film thickness. We crudely approximate our screened electron-electron interaction by a dipole interaction, $(2 e t)^{2} / r^{3}$. The energy density is given approximately as [11]

$$
u=4 n e^{2} t^{2} / \pi a L^{2}-W n_{t}^{1 / 2} / L
$$

Here $n$ is the density of electrons, a is the lattice spacing, $W$ is the impurity potential, and $n_{i}$ is the density of impurity potentials. The coherence length $L$ is obtained by minimizing the energy with respect to $L$. The threshold field is estimated by setting the energy gained by moving the crystal one lattice spacing to the cost in impurity energy [12]. We obtain L $\sim 10^{3}$ a for our sample. This suggests that our electron solid is very weakly pinned. 


\section{Discussion.}

The conduction and noise features exhibited by the pinned electron solid mimic those of sliding charge-density waves and have a simple interpretation. At low excitation fields the ac conductivity is due to small amplitude oscillations of lattice domains in the impurity potential wells. Above the lower critical voltage the solid moves by the uncorrelated depinning of individual domains. This leads to $a \mathrm{f}^{-\alpha}$ noise spectrum. Above a second critical voltage $V_{N}$ the entire solid becomes depinned, and the resistivity is determined by momentum transfer between lattice phonons and ripplons and from the scattering of the solid from the random impurity potentials. In this regime the large broad-band noise results from the lattice "jerking" over the pinning sites. A frequency dependence of the form $\omega^{-\eta}$ can be obtained by a distribution of impurity potentials with a uniform distribution of resonance frequencies extending to zero frequency.

The conduction and noise characteristics of other pinned two-dimensional electron solids can be compared with our results. Kajita [1] in his study of the classical electron lattice on a helium film above a hydrogen substrate plots a set of I-V characteristics for different electron densities. The electron density is proportional an electric field applied normal to the electron layer. This electric potential is another source of trapping on a rough hydrogen surface. He finds continuous $I-V$ characteristics at the critical field although at the highest density, largest pinning potentials, there is an apparent step in the $\mathrm{I}-\mathrm{V}$ curve at the critical field. The data points are discrete. He uses arbitrary units for the applied fleld, and one cannot extract a domain size from his data.

Goldman et al. [4] find a differential resistance which varies smoothly with applied voltage. Their curves resemble the data shown in Fig. 2, with a large separation between $V_{c}$ and $V_{N}$. Their lattice is also weakly pinned with a domain size of $\mathrm{L}=250 \mathrm{a}$. Li et al. [6] and Pudalov et al. [7] find a sharp variation of dynamic resistance with voltage as compared to either our data or the data of Goldman et al. Li et al. find a domain size of $<100$ a and $L$ is much less in the MOSFET sample. These solid phases are more strongly pinned. Williams et al. [5] find a large, discrete step in the I-V characteristics at a critical field which is two orders of magnitude larger than the critical fields measured in the heterostructures of Refs. 4 and 6 . They obtain a domain size of $\sim 20$ a using this critical field as the depinning field. Jiang et al. [13] have reproduced both the dynamical resistance curves of Goldman et al. at low voltages and this step in the I-V characteristics seen by Williams et al. at a higher threshold voltage on the same sample. They find the threshold power to be independent of fllling factor. They argue that this result is consistent with high-field breakdown caused by excessive electron heating.

The authors of Refs. 4,6 and 7 find a large increase in the broad-band noise which sets in when the dynamical resistance vanishes, i.e. when the lattice becomes completely depinned.

A nonlinear dynamic resistance has also been reported in the insulating phase of a two-dimensional hole system [15]. These characteristics are qualitatively different than the results shown in Fig. 2.

\section{Conclusions.}

Measurements on electrons supported by a helium film yield exceptionally clear features of the conduction and noise characteristics of a pinned electron solid. A consistent interpretation of the data is the following. A weaklypinned electron crystal is characterized by a dynamic resistance which is a smooth function of the applied fleld with a large range between the critical field for the depinning of domains and the critical field for the onset of broad-band noise. The strongly-pinned electron solid is characterized by a dynamic resistance which is a sharp function of applied field above the critical field. Sufficlently strong pinning might lead to a discrete step in 
the I-V characteristics. The similarity of the conduction and noise features in quantum systems and those of the classical pinned electron solid provides supporting evidence that the electron solid has been observed in these quantum systems.

The electron on hellum system has a number of advantages for the study of the pinned solid phase. The pinning potential can be varied, in situ, by changing the helium film thickness, the electron density can be easily adjusted and the type of pinning potential can be altered by depositing charges on the underlying substrate [14]. This includes both strongly repulsive interactions with trapped negative charges and positive charges which trap single electrons in the solid.

\section{References.}

[1] For reviews, see: Gruner G. and Zettl A., Phys. Rep. 119 (1985) 117, and Gruner G., Rev. Mod. Phys. 60 (1988) 1129.

[2] Kajita K., Jpn. J. Appl. Phys. 25, Suppl. 3 (1987) 1943; Surf. Sci. 196 (1988) 29.

[3] Jiang H.W. and Dahm A.J., Phys. Rev. Lett. 62 (1989) 1396.

[4] Goldman V.J., Santos M., Shayegan M. and Cunningham J.E., Phys. Rev. Lett. 65 (1990) 2189.

[5] Williams F.I.B., Wright P.A., Clark R.G., Andrei E.Y., Deville G., Glattli D.C., Probst O., Etlenne B., Dorin C., Foxon C.T. and Harris J.J., Phys. Rev. Lett. 66 (1991) 3285.

[6] Li Y.P., Sajoto T., Engel L.W., Tsui D.C. and Shayegan M, Phys. Rev. Lett. 67 (1991) 1630.

[7] Pudalov V.M., D' Iorio M., Kravchenko S.V. and Campbell J.W., Phys. Rev. Lett. 70 (1993) 1866.

[8] Mehrotra R. and Dahm A.J., J. Low Temp. Phys. 67 (1987) 115.

[9] Saitoh M., Phys. Rev. B40 (1989) 810.

[10] Jiang H.W. and Dahm A.J., Surf. Sci. 229 (1990) 352.

[11] Fukuyama H. and Lee P.A., Phys. Rev. B17 (1978) 535.

[12] Lee P.A. and Rice T.M., Phys. Rev. B19 (1979) 3970.

[13] Jiang H.W., Stormer H.L., Tsui D.C., Pfeiffer L.N. and West K.W., Phys. Rev. B44 (1991) 8107.

[14] Ruzin I.M., Marianer S. and Shklovskii B. I., Phys. Rev. B46 (1992) 3999.

[15] Santos M.B., Suen Y.W., Shayegan M., Li Y.P., Engel L.W. and Tsui D.C., Phys. Rev. Lett. 68 (1992) 1188. 